\title{
DELAY-BASED SIGNAL SHAPERS AND ACFA 2020 BLENDED WING BODY FLIGHT CONTROL SYSTEM
}

\author{
V. Kucera ${ }^{1}$ and M. Hromčík ${ }^{2}$ \\ ${ }^{1}$ Czech Technical University in Prague, FEE \\ Department of Control Engineering \\ Prague, Czech Republic \\ ${ }^{2}$ Czech Technical University in Prague, FEE \\ Center for Applied Cybernetics \\ Prague, Czech Republic
}

\begin{abstract}
The purpose of this paper is twofold. First: results related to application of signal shapers, imposed on pilot's commands, in cooperation with feedback flight control system (FCS) are reported for the case of ACFA2020 (Active Control for Flexible 2020 Aircraft) blended-wingbody (BWB) design. The results suggest that signal shapers can cooperate nicely both with FCS focused on the rigid-body dynamics only, as well as with an implemented and properly working active damping system. In both cases, the amount of vibrations due to pilot's inputs (manoeuvres) can be substantially reduced. Second: combination of signal shapers and rate-limiters is discussed in detail. Rate-limiters, representing finite achievable rates of servos for control surfaces, deteriorate considerably performance of the delay-based shapers. Configuration proposes only open-loop response of the free aircraft (without controller) for shaped reference respect to nonlinearities at action surface. Standard versions of the shapers cannot be therefore directly applied, especially for higher control surfaces deflections. Instead, two efficient alternatives can be used, suggested in the paper, that take the rate limitations into account at the design stage already.
\end{abstract}

\section{SIGNAL SHAPERS}

Signal shapers (PosiCast, zero vibration (ZV), ZV derivative (ZVD), extra insensitive (EI), etc.) have been in the center of attention for decades [1-3], as an efficient feedforward control tool for manipulation of oscillatory systems, like 
large cranes, lightweight (therefore, flexible) manipulators, or mechanical structures [4-6]. Signal shaping is implemented by convolving a sequence of impulses, an input shaper, with any desired command. The shaped command that results from the convolution is then used to drive the system. If the impulses in the shaper are chosen correctly, then the system will respond without vibration to any unshaped command.

\section{SIGNAL SHAPERS AND FLIGHT CONTROL SYSTEM FOR FLEXIBLE AIRCRAFT}

Apparently, signal shapers are clear candidates for inclusion into an efficient FCS for flexible aircraft like the ACFA 2020 BWB design (www.acfa2020.eu) (Fig. 1).

For some reason though, signal shapers are not commonly known in the flight controls area and some more traditional solutions like "structural filters" are routinely used - basically, low-pass Chebyshev or other-type filters included in the FCS so as not to excite flexible modes. In comparison, properly tuned signal shapers, targeted at the most prominent flexible modes of the aircraft, lead to superior responsiveness and more efficient vibrations suppression.

The role and placement of a properly designed PosiCast shaper in a traditional feedback Stability Augmentation System (SAS) or Control Augmentation System (CAS) is depicted in Fig. 2; SAS/CAS is not supposed to act as flexible modes damper.

For a SAS/CAS augmented by (or integrated with) feedback active damping system, the scheme changes as in Fig. 3 (for a particular case of roll autopilot).

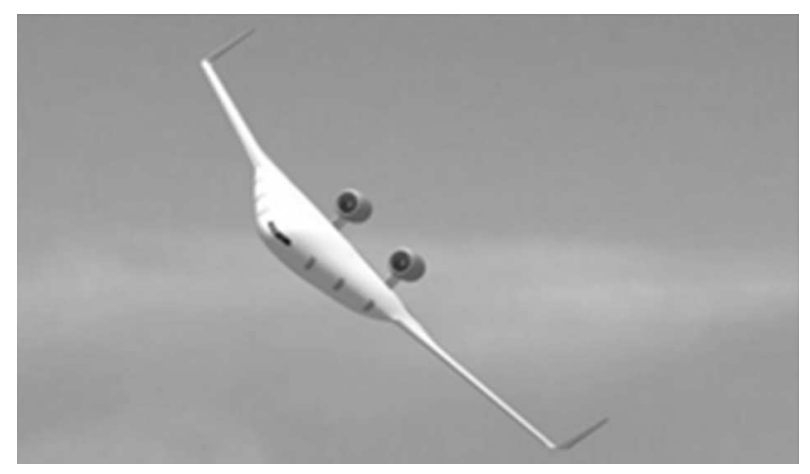

Figure 1 The ACFA2020 BWB aircraft 
... PosiCast \& SAS

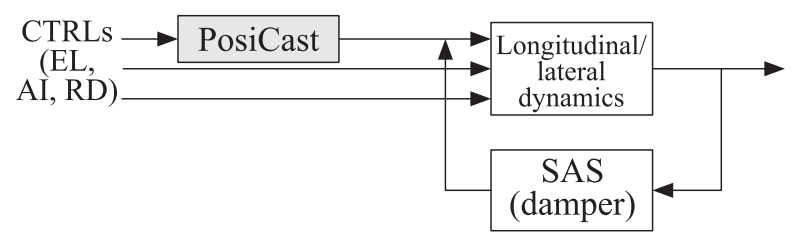

... PosiCast \& CAS
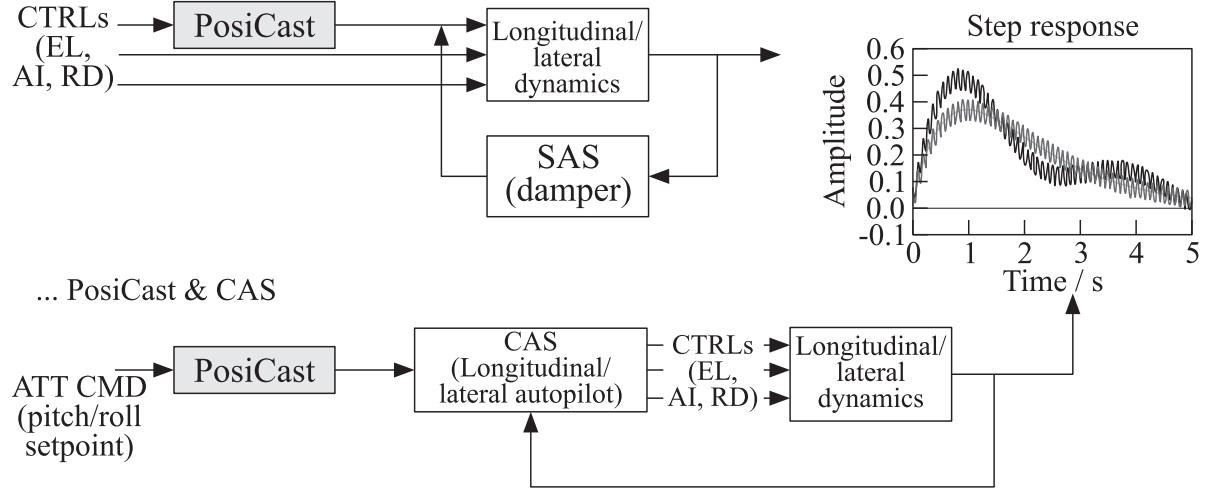

Figure 2 Placement and function of PosiCast input command shaper in a feedback SAS/CAS

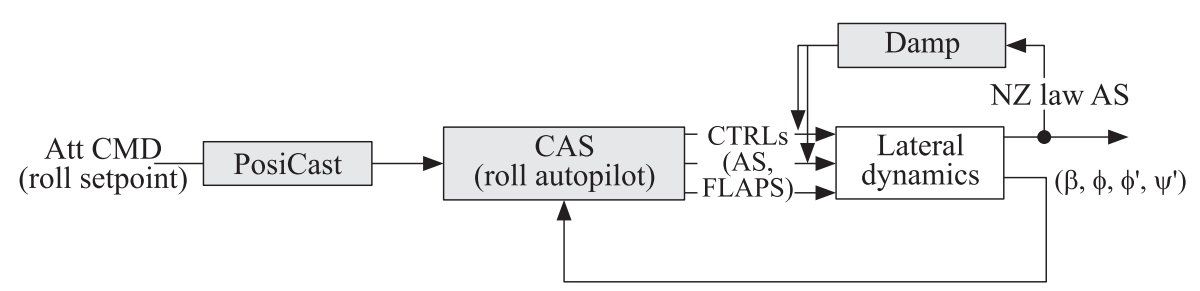

Figure 3 PosiCast and active-damping-augmented lateral CAS

\section{RESULTS FOR ACFA2020 BLENDED-WING-BODY AIRCRAFT}

First, for the purpose of this paper, a very simple SAS (simple input simple output (SISO), stabilizing unstable longitudinal dynamics) is considered and classical PosiCast signal shaper is included in the stick-input channel. The aircraft dynamics is left as unchanged as possible obviously.

Transfer function from stick input to the wings modal sensor (accelerometerbased, Nz law) is in Figs. 4-6, showing significant damping of wings first two flexible symmetric modes (red), compared to a free aircraft (green) and also to the Chebyshev-type structural low-pass filter (blue). Note that a two-modes (four substeps) version of PosiCast was designed to cover both modes simultaneously: all 18 mass cases (6 for fuel, 3 for passengers). 


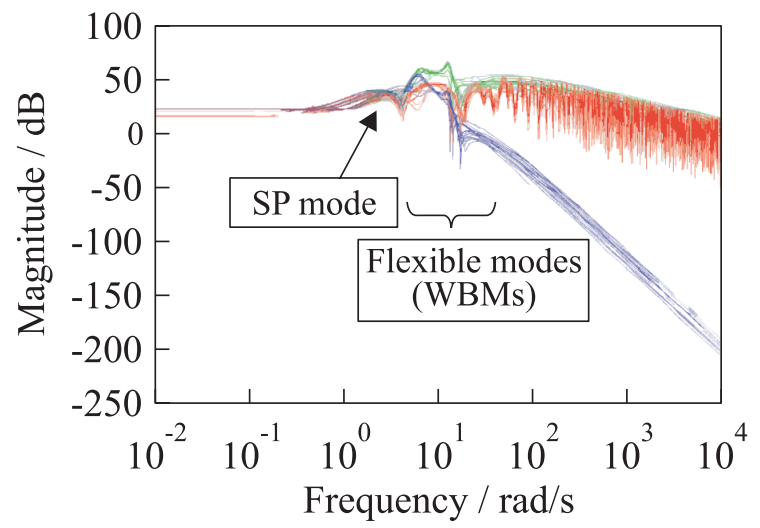

Figure 4 PosiCast and longitudinal NACRE model. Stick to NZ law wings (bode). Damping of wings first two flexible symmetric modes by PosiCast (red), Chebyshevtype structural low-pass filter (blue), and frees aircraft (green).

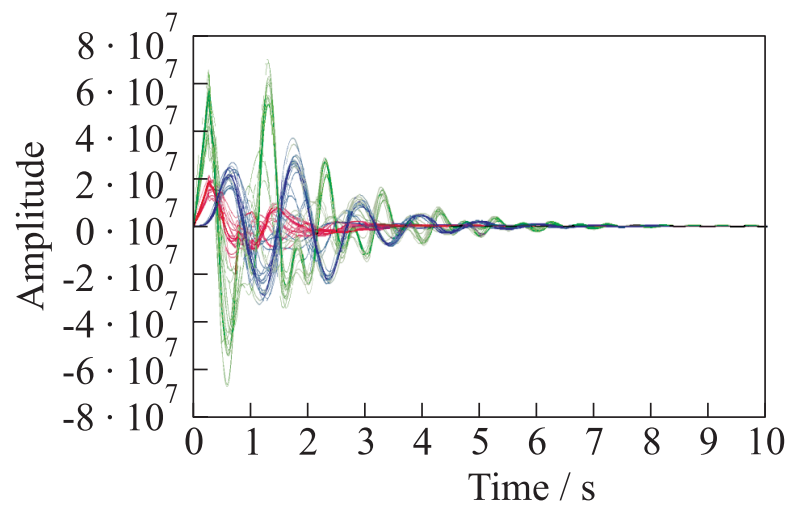

Figure 5 PosiCast and longitudinal control. Stick to modal sensor. Reference command (step) filtered by PosiCast (red), Chebyshev-type structural low-pass filter (blue), and frees aircraft (green).

The PosiCast shaper certainly affects responsiveness of the aircraft (see the close coupling of flexible and short period (SP) modes). In any case nevertheless, it does not affect it more negatively than the structural filter (Fig. 6).

In this case, PosiCast is acting directly on the control surface signals where the rate limiters need to be taken into account. All effects described in section 4 are evidenced (PosiCast out of the game for step stick command and elevator deflection above $5^{\circ}$ ) and the measures proposed in the next sections (like ramp 


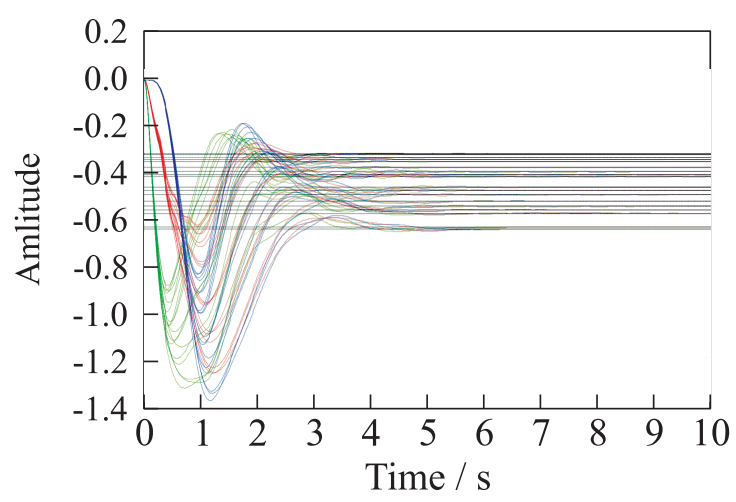

Figure 6 PosiCast and longitudinal NACRE model. Stick to $q$ (step). Performance of PosiCast (red), Chebyshev-type structural low-pass filter (blue), and frees aircraft (green).

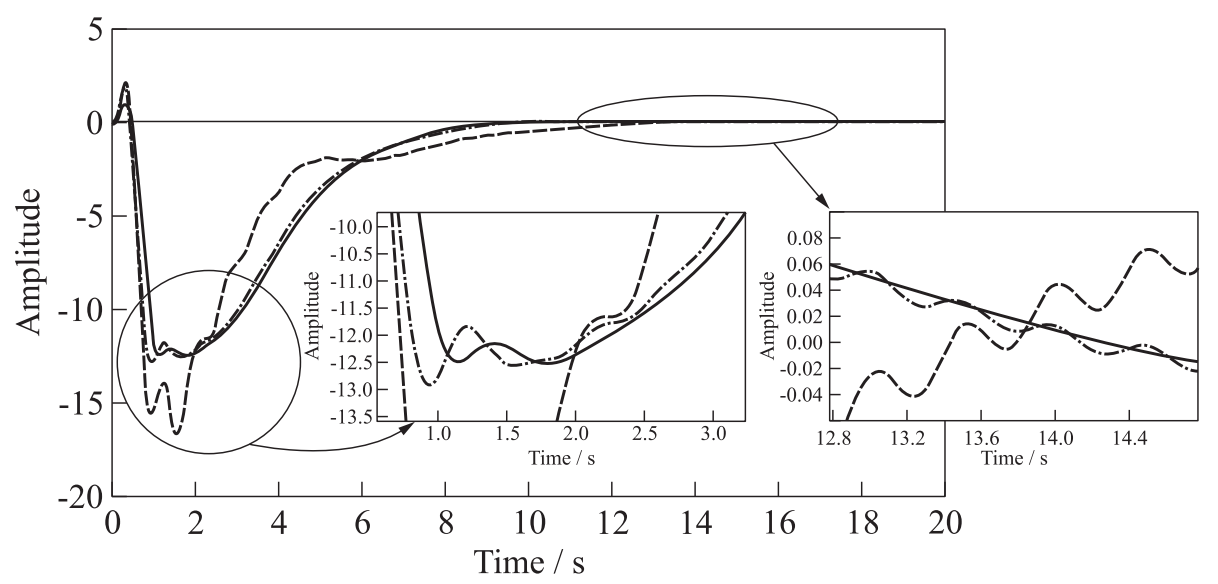

Figure 7 Blended wing body lateral autopilot (dashed curves), augmented with active FB damper (dash-dotted curves), and with PosiCast on top of that (solid curves); roll SP to NZ law wings antisymmetric (step); selected passengers-fuel combination: $2-5$

split-up for higher amplitudes) lead to exactly the same results (refer to section 4 for detailed description).

Further results for both longitudinal and lateral ACFA2020 BWB controls, also in combination with active damping feedback system are shown in Fig. 7.

Compare the modal sensor reading (lateral CAS, step for roll-angle set point), for FCS only (dashed curves), FCS + feedback active damper (dash-dotted 


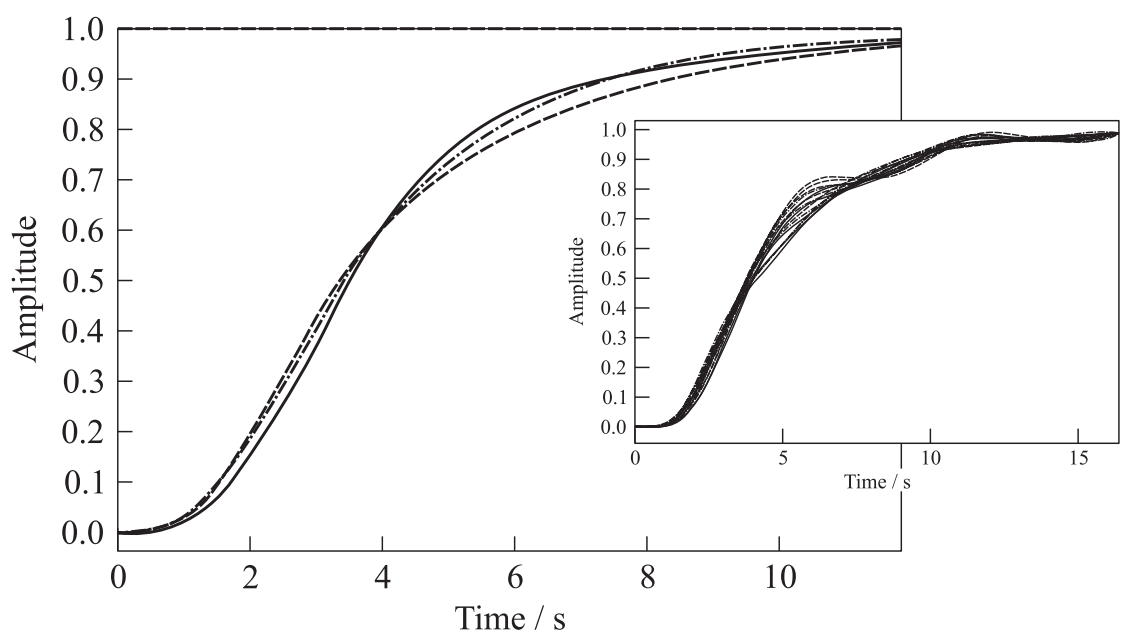

Figure 8 Blended wing body lateral autopilot (dasged curves), augmented with active FB damper (dash-dotted curves), and with PosiCast on top of that (solid curves); roll SP to roll Angle (step); selected passengers-fuel combination: 2-5, all cases (subfigure)

curves), and with PosiCAST shaper on top of that all. Contribution of the signal shaper in this setup, for reduction of vibrations caused by the automatically engaged manoeuvre, is obvious.

In addition, responses of the aircraft in all cases are almost identical (Fig. 8).

\section{SIGNAL SHAPERS AND RATE LIMITERS}

As shown, delay-based input shaper, like ZV and EI, can be effectively used as a feedforward reference filter applied to pilot command in order to reduce wing bending and vertical bending of hull during a maneuver.

This strategy is, nevertheless, strongly limited by the rate limiter nonlinearity (standing for finite servos rates), having substantial, amplitude-dependent filtering effect on the input signal. This observation can be interpreted both in the frequency and time domain terms. Speaking in frequency-domain words, rate limiter acts as a low-pass filter, with cut-off frequency strongly dependent on the amplitude of the input signal (Fig. 9). The higher the amplitude is, the stronger filtering effect arises. By inspection of the dependency in Fig. 9, for elevator commands greater than $5^{\circ}$ (cut-off approximately $15 \mathrm{rad} / \mathrm{s}$ for five degrees amplitude), the influence of the delay-based filters on the command signal is strongly weakened by the rate limiter at the higher frequency of the hull bending mode 


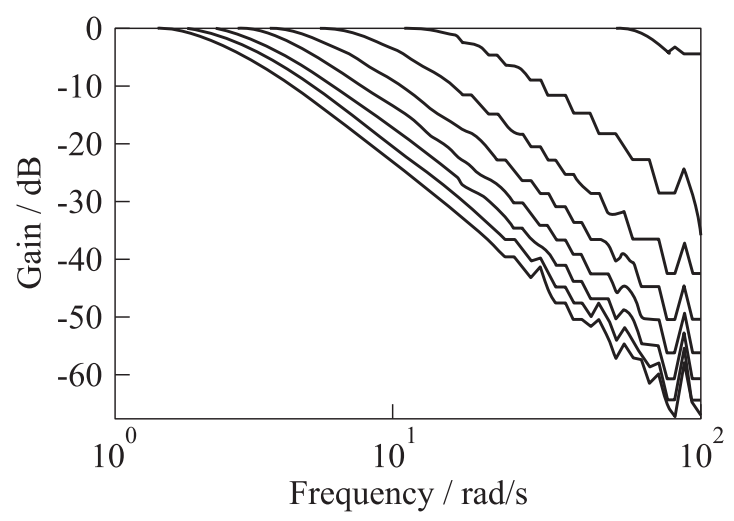

Figure 9 Frequency responses of a $30 \mathrm{deg} / \mathrm{s}$ rate limiter for varying signal amplitude

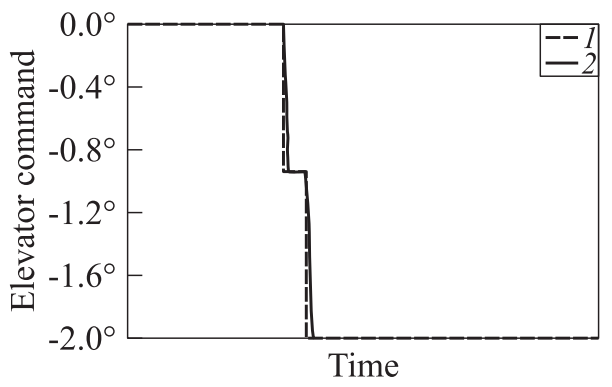

(a)

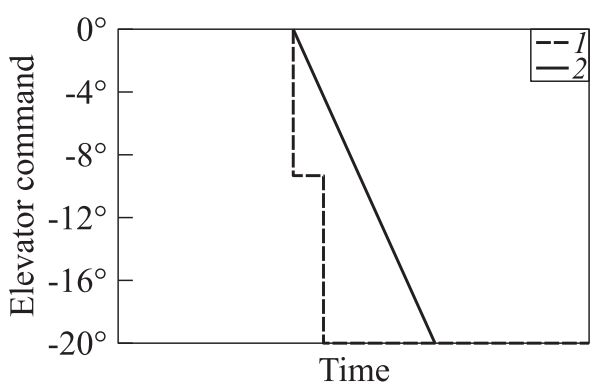

(b)

Figure 10 Effect of rate limiter for elevator command amplitude of $2^{\circ}(a)$ and $20^{\circ}(b)$ : 1 - rate limiter effect; and 2 - input

(HBM). Considering these facts, filters cannot, in principle, be successful for all cases. From the time-domain perspective, the deterioration that rate-limiters cause to the reference shaped step command, can be also nicely demonstrated in Fig. 10. Compare these two pictures, for elevator command amplitude of $2^{\circ}$ and $20^{\circ}$, respectively - output of the preshaper (1), when passed subsequently through rate-limiter of $30 \mathrm{deg} / \mathrm{s}(2)$.

For this reason, two approaches are suggested. In both cases, the shaped signal is modified in such a way that it becomes tractable through the subsequent rate-limiter block without distortion (unlike the pure signal shaper output itself).

The first approach is based on splitting the ramp signal, coming out from a rate-limiter block as a response to step, artificially, for a time-delay slightly 


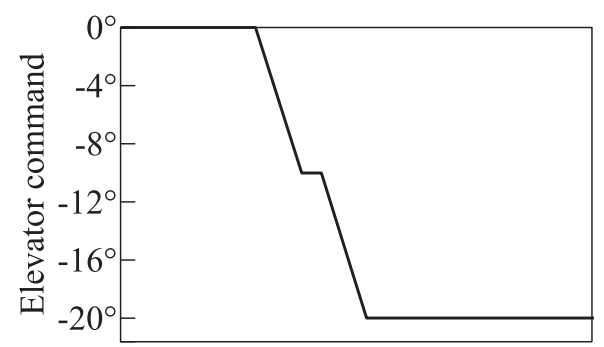

Time
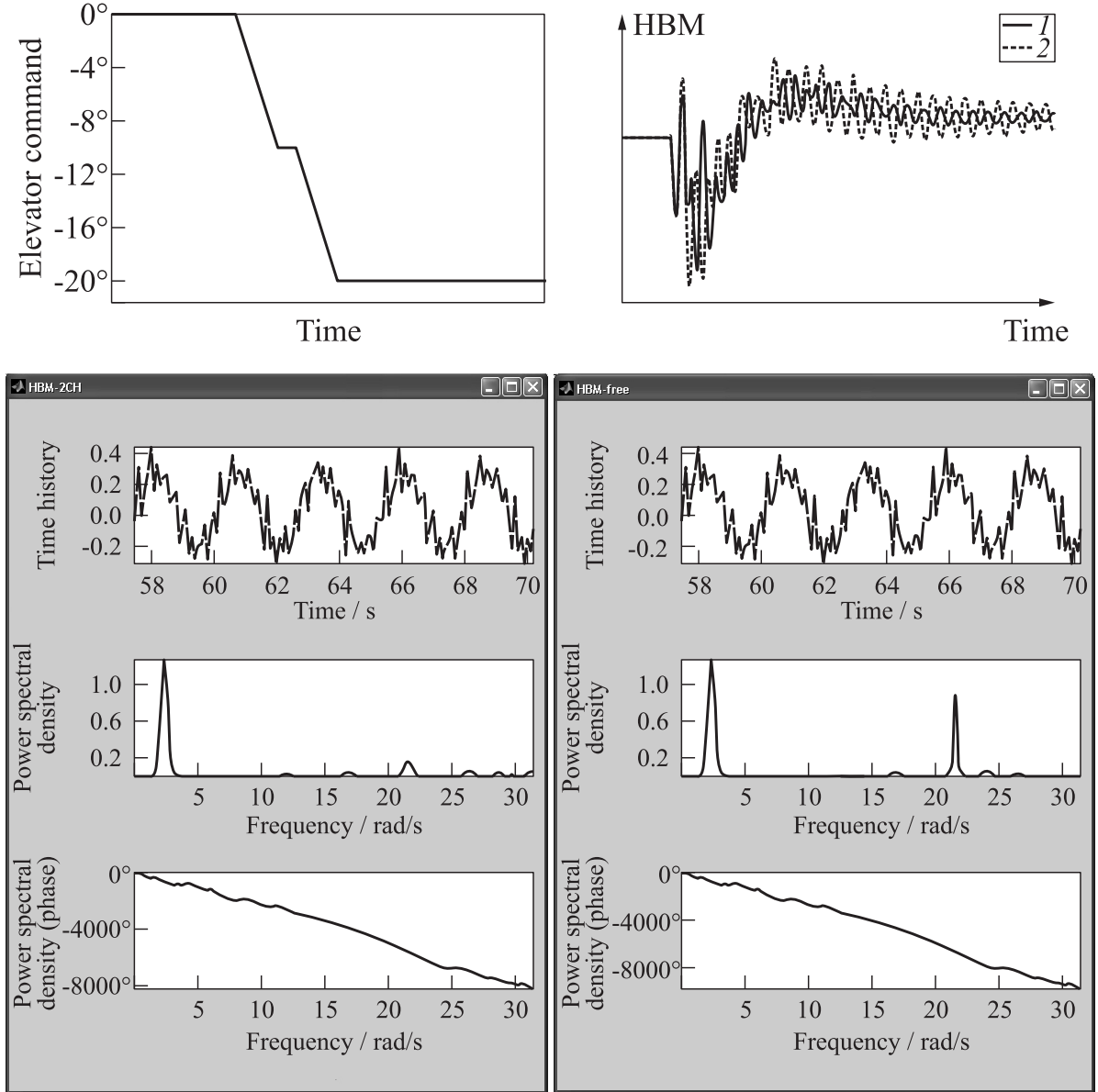

Figure 11 Modified shaper and effect on HBM sensor. Power spectral density of HBM sensor with modified shaper corresponds to HBM-2CH (1) and without modified shape to HBM-free (2)

smaller than the ZV shaper-suggested value. This leads to a fair reduction of the HBM peak (Fig. 11). In this particular case, the shaped reference in Fig. 11 for the 20 degree elevator command, which has transfer function:

$$
\begin{aligned}
& G_{\text {mod }}=\frac{1}{\text { spoint }}\left(\frac{\text { rate }}{s} e^{- \text {spoint } \cdot \text { rate } / 2}\right)+\left(\frac{\text { rate }}{s}\right.\left.e^{-(\text {spoint } \cdot \text { rate } / 2+\text { delay }) s}\right) \\
&-\left(\frac{\text { rate }}{s} e^{-(\text {spoint } / \text { rate }+ \text { delay }) s}\right)
\end{aligned}
$$




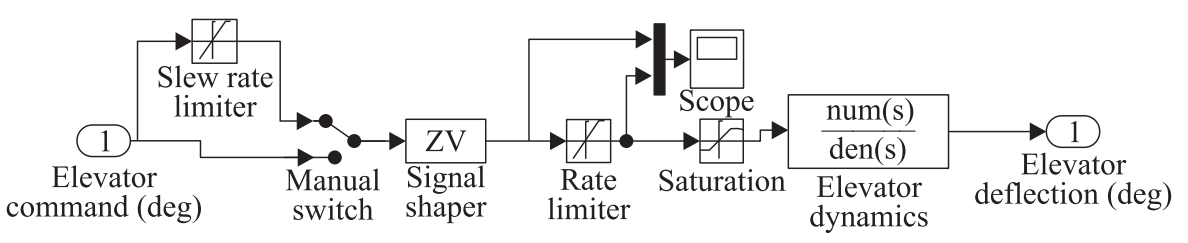

Figure 12 Configuration of elevator model interconnected with signal shaper as open loop filter of reference command

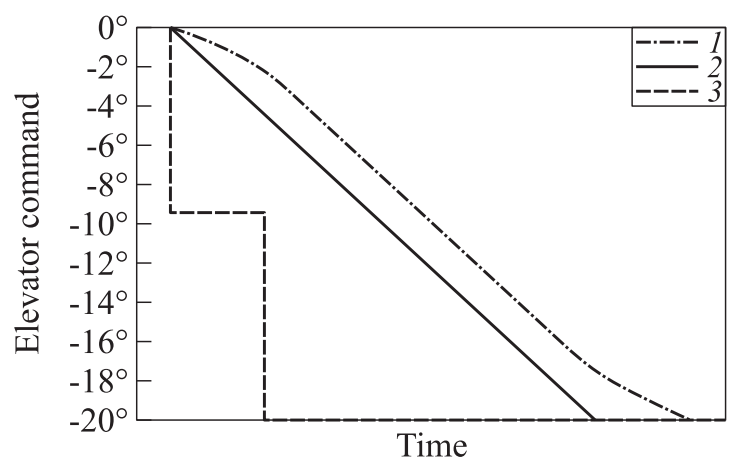

Figure 13 Effect of the rate limiter for shaped reference command: 1 - improved command; 2 - rate limiter effect; and 3 - input

where 'spoint' is the set point; 'rate' is the setting of rate limiter; and 'delay' is the value from PosiCast approach.

This command is fully accepted by rate limiters without any distortion. The filter $G_{\text {mod }}$ is, unfortunately, parameterized by the amplitude of the step; so, it is not a constant or time-invariant system.

Alternatively, the following procedure can be applied. The main idea is to attach an additional rate limiter, with the same setting as the one representing servos, in front delay-based shaper (Fig. 12). The modified filtered command is obviously accepted by (passed-through) the finite-rate servos without any distortion (line 1 in Fig. 13), and it does not contain frequencies corresponding to flexible modes of aircraft (as the signal shaper is in the command line). The results of the hull bending sensors in Fig. 14 show power of this method, where curve 2 is not treated by new approach and curves 1 (ZV) and 2 (EI) are for different used shapers with naturally adaptive rate limiter before. 


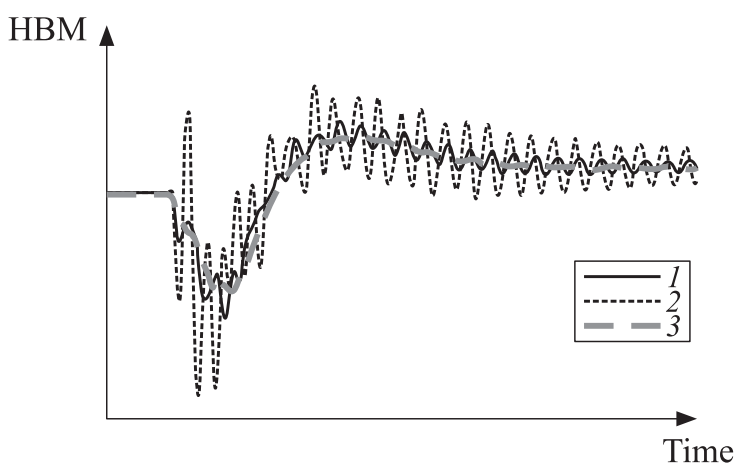

Figure 14 Bending of hull for $20^{\circ}$ of reference command: 1 - improved ZV shaper and rate limiter; 2 - without shapers; and 3 - improved EI shaper and rate limiter

\section{CONCLUDING REMARKS}

Results will be further developed and applied for the case study of large flexible BWB aircraft, similarly to section 3 (for the classical signal shapers designs). Data come from the ongoing European project ACFA2020. Active Control for Flexible Aircraft (ACFA2020, www.acfa2020.eu) is a collaborative research project funded by the European Commission under the 7th research framework programme (FP7). The project deals with innovative active control concepts for ultraefficient 2020 aircraft configurations like the BWB aircraft.

The results suggested in section 2 and evaluated in section 3 show signal shapers cooperation with FCS focused on the rigid-body dynamics only, as well as with an implemented and properly working active damping system. This approach can be efficient robust alternatives to classical low-pass filters as assistance for a feedback system.

The results in section 4 deal with problem of finite rate of action surface. Two extended strategies of classical signal shapers are presented for a case of free aircraft dynamics without feedback configuration. Modification respects to nonlinearities keep filtering effect of signal shapes.

\section{REFERENCES}

1. Smith, O. J. M. PosiCast control of damped oscillatory systems. 1957. Proceedings of the IRE. Col. 45:1249-55.

2. Singer, N. C., and W.P. Seering. 1990. W.P. Preshaping command input to reduce system vibration. J. Dyn. Syst. Measure Control 112(1):76-82. 
3. Singhose, W.E., S. Derezinski, and N.C. Singer. 1996. Extra-insensitive input shapers for controlling flexible spacecraft. J. Guidance Control Dyn. 19(2):285-391.

4. Singhose, W.E., S. Derezinski, and N.C. Singer. 1996. Extra-insensitive input shapers for controlling flexible spacecraft. J. Guidance Control Dyn. 19(2):285-391.

5. Li, B., X. Zhang, J. K. Mills, W. L. Cleghorn, and Liyang Xie. 2009. Vibration suppression of a 3-PRR flexible parallel manipulator using input shaping. Conference (International) on Mechatronics and Automation. China.

6. Ahmed, M. A., M. S. Ramli, R. M. T. Raja Ismail, N. Hanbali, and M. A. Zawawi. 2009. The investigation of input shaping with optimal state feedback for vibration control of flexible joint manipulator. Conference on Innovative Technologies in Intelligent Systems and Industrial Applicaton. Malaysia. 
Figure 4 PosiCast and longitudinal NACRE model. Stick to NZ law wings (bode). Damping of wings first two flexible symmetric modes by PosiCast (red), Chebyshevtype structural low-pass filter (blue), and frees aircraft (green). (Refer Kucera and Hromčík, p. 770.)

Figure 5 PosiCast and longitudinal control. Stick to modal sensor. Reference command (step) filtered by PosiCast (red), Chebyshev-type structural low-pass filter (blue), and frees aircraft (green). (Refer Kucera and Hromčík, p. 770.)

Figure 6 PosiCast and longitudinal NACRE model. Stick to $q$ (step). Performance of PosiCast (red), Chebyshev-type structural low-pass filter (blue), and frees aircraft (green). (Refer Kucera and Hromč́́k, p. 771.) 